\title{
Serous tubal intraepithelial carcinoma upregulates markers associated with high-grade serous carcinomas including Rsf-1 (HBXAP), cyclin E and fatty acid synthase
}

\author{
Ann Smith Sehdev ${ }^{1}$, Robert J Kurman², Elisabetta Kuhn² and Ie-Ming Shih ${ }^{2}$ \\ ${ }^{1}$ Department of Pathology, Legacy Health Systems, Portland, OR, USA and ${ }^{2}$ Department of Pathology, Johns \\ Hopkins Medical Institutions, Baltimore, MD, USA
}

\begin{abstract}
Serous tubal intraepithelial carcinoma (STIC) has been proposed as a precursor for many pelvic high-grade serous carcinomas. Our previous analysis of the ovarian cancer genome identified several genes with oncogenic potential that are amplified and/or overexpressed in the majority of high-grade serous carcinomas. Determining whether these genes are upregulated in STICs is important in further elucidating the relationship of STICs to high-grade serous carcinomas and is fundamental in understanding the molecular pathogenesis of high-grade serous carcinomas. In this study, 37 morphologically defined STICs were obtained from 23 patients with stage IIIC/IV high-grade serous carcinomas. Both STICs and the high-grade serous carcinomas were analyzed for expression of Rsf-1 (HBXAP), cyclin E, fatty acid synthase (FASN) and mucin-4. In addition, they were examined for expression of established markers including p53, Ki-67 and p16. We found that diffuse nuclear p53 and p16 immunoreactivity was observed in $27(75 \%)$ of 36 and $18(55 \%)$ of 33 STICs, respectively, whereas an elevated Ki-67 labeling index ( $\geq 10 \%)$ was detected in $29(78 \%)$ of 37 STICs. Cyclin E nuclear staining was seen in $24(77 \%)$ of 35 STICs, whereas normal tubal epithelial cells were all negative. Increased Rsf-1 and FASN immunoreactivity occurred in $63 \%$, and $62 \%$ of STICs, respectively, compared with adjacent normal-appearing tubal epithelium. Interestingly, only one STIC showed increased mucin-4 immunoreactivity. Carcinomas, when compared with STICs, overexpressed p16, Rsf-1, cyclin E and FASN in a higher proportion of cases. In conclusion, STICs express several markers including Rsf-1, cyclin E and FASN in high-grade serous carcinomas. In contrast, mucin-4 immunoreactivity either did not change or was reduced in most STICs. These results suggest that overexpression of Rsf-1, cyclin E and FASN occurs early in tumor progression.
\end{abstract}

Modern Pathology (2010) 23, 844-855; doi:10.1038/modpathol.2010.60; published online 12 March 2010

Keywords: ovarian cancer; tubal intraepithelial carcinoma; HBXAP (Rsf-1)

Characterization of putative precursor lesions is fundamental in elucidating the molecular pathogenesis of cancer and has profound implications for early detection, prevention and treatment. In contrast to other major types of carcinomas including colorectal, prostate, pancreatic and breast cancer, the cell origin of ovarian cancer has not yet been identified despite numerous studies that have carefully examined the ovaries for precursor lesions.

Correspondence: Professor Ie-Ming Shih, MD, PhD, Department of Pathology, Johns Hopkins Medical Institutions, 1550 Orleans Street, CRB-2, Rm: 305, Baltimore, MD 21231, USA.

E-mail: shihie@yahoo.com

Received 02 December 2009; revised 4 January 2010; accepted 5 January 2010; published online 12 March 2010
Recent histopathological studies, however, have provided provocative new evidence that the fallopian tube, specifically the fimbria, rather than the ovarian surface epithelium may be the source of most ovarian high-grade serous carcinomas. ${ }^{1}$ The basis for this hypothesis comes from morphologic, immunohistochemical and molecular genetic studies of a lesion designated 'serous tubal intraepithelial carcinoma' (STIC), which closely resembles high-grade ovarian serous carcinoma and coexists with small, early invasive tubal carcinomas in women with a genetic predisposition to ovarian cancer. $^{2,3}$ In addition, STICs are also detected in prophylactic specimens of fallopian tubes from women who do not have an associated carcinoma. ${ }^{4}$ More recently, Kindelberger et $a l^{5}$ have reported that 
over $70 \%$ of sporadic (nonhereditary) ovarian and peritoneal high-grade serous carcinomas displayed mucosal tubal involvement by carcinoma including STICs, suggesting that the latter are potential precursors for sporadic as well as hereditary highgrade serous carcinomas. Further support for the tubal origin of high-grade ovarian serous carcinoma comes from the finding of strong, diffuse p53 immunoreactivity in the majority of STICs $^{4}$ as well as the detection of identical TP53 mutations in the STICs and concurrent ovarian serous carcinomas, indicating a clonal relationship between them. ${ }^{5}$

Moreover, STICs with TP53 mutations have also been detected in the absence of a concomitant ovarian serous carcinoma, suggesting that STICs precede the development of high-grade serous carcinoma rather than representing a metastasis from a primary ovarian carcinoma. Finally, a gene profiling study shows that serous carcinomas from the fallopian tube and ovary are indistinguishable ${ }^{6}$ and that the expression profile of high-grade serous carcinoma is more closely related to the fallopian tube than to ovarian surface epithelium. ${ }^{7}$

To further characterize the molecular changes in STICs, we analyzed them for gene expression using several well-established ovarian cancer-associated genes including Rsf- $1,{ }^{8}$ cyclin $\mathrm{E},{ }^{9}$ fatty acid synthase $(\text { FASN })^{10}$ and mucin- $4^{11}$ that are frequently amplified and/or upregulated in high-grade serous carcinoma. As STICs are almost always incidental microscopic findings detected during histopathology review, it is very difficult to harvest sufficient fresh tissue from STICs to perform mRNA-based gene expression analysis. We therefore applied immunohistochemistry on paraffin sections using well-characterized antibodies to study gene expression in STICs. We found that all the selected genes except mucin-4 were upregulated in most STICs when compared with adjacent normal-appearing tubal epithelium. The frequency of overexpression is similar to that found in a large series of high-grade serous carcinoma that has been reported earlier. ${ }^{9,12,13}$ Our results provide additional evidence that STICs are the likely precursors of high-grade serous carcinomas and suggest that overexpression of Rsf-1 (HBXAP), cyclin E and FASN occurs at an early stage in tumor development.

\section{Materials and methods}

\section{Case Selection}

The criteria for case selection were based on the lesions exhibiting three or more of following histologic features including (1) abnormal chromatin pattern, (2) nuclear enlargement, (3) marked pleomorphism, (4) epithelial stratification and/or loss of polarity and (5) nuclear molding. Thirtyseven morphologically defined STICs were obtained from 23 patients with stage IIIC/IV high-grade serous carcinoma collected from the Johns Hopkins Hospital. Many of the specimens were sent as consultation cases from Legacy Health Systems, Portland, Oregon. Tissue collection conformed to the guidelines of the Institutional Research Board of the Johns Hopkins Hospital.

\section{Immunohistochemistry}

Both STICs and the high-grade serous carcinomas were analyzed for expression of ovarian cancerassociated markers including Rsf-1 (HBXAP), FASN, cyclin $\mathrm{E}$ and mucin-4. These four proteins were selected because they are expressed in a high proportion of high-grade serous carcinomas. In addition, STICs and high-grade serous carcinoma were examined for expression of 'conventional' markers including p53, Ki-67 and p16. The sources and dilution for each antibody were: Rsf-1 antibody (Millipore Upstate Cell Signaling, Billerica, MA, USA; 1:2000 dilution), FASN antibody (FASgen, Baltimore, MD, USA; 1:50 dilution), cyclin E antibody (Zymed, Invitrogen; 1:250 dilution), mucin-4 antibody (Abcam, Cambridge, MA, USA; 1:1500 dilution), p53 antibody (Ventana, Tucson, AZ, USA; 1:1 dilution), Ki-67 antibody (Ventana; 1:1 dilution) and p16 antibody (Cintec, Kirkland, WA, USA; 1:1 dilution).

Antigen retrieval was performed by steaming the sections in citrate buffer ( $\mathrm{pH}$ 6.0) for $20 \mathrm{~min}$. After incubation with the primary antibodies at room temperature for $2 \mathrm{~h}$, a positive reaction in tissue sections was detected by the EnVision + System (DAKO, Carpinteria, CA, USA) and developed with 3,3'-diaminobenzidine. The percentage of intensely immunoreactive nuclei were determined for p53, Ki-67, p16 and cyclin E, whereas a three-tier intensity score was used to compare the immunoreactivity of Rsf-1, FASN and mucin-4 in STICs to adjacent normal-appearing tubal epithelium. This grading system for Rsf-1, FASN and mucin-4 was used because the normal fallopian tube epithelium expresses a low level of these markers. Accordingly, the final intensity score for these markers was derived by subtracting the intensity of the normal tubal epithelium from the initial intensity score of the STIC in the same specimen. In the process of cutting sections for immunohistochemistry, some of the STICs were exhausted and therefore not all of them could be evaluated for all of the markers.

\section{Results}

Thirty-seven STICs from 23 patients were analyzed. All were flat lesions except for three that exhibited a papillary architecture (patient 7 , patient 9 and STIC-1 in patient 23). Twenty-two (59.4\%) STICs were located in the fimbriated end of the tubes. 
Multiple STICs were detected in eight patients. Twelve high-grade serous carcinomas co-existed with STICs in the same tissue sections. The morphologic features of the STICs and associated ovarian carcinomas were similar. The expression of all markers in STICs and high-grade serous carcinomas using immunohistochemistry is summarized in Table 1 and is discussed below. p53

Diffuse nuclear p53 ( $\geq 50 \%$ of nuclei) immunoreactivity was observed in $27(75 \%)$ of 36 STICs (Figure 1) and defined as intense immunoreactivity. In contrast, the normal-appearing tubal epithelium adjacent to the STICs did not show detectable nuclear p53 immunoreactivity except in the

Table 1 Immunostaining results in all TICs and associated high-grade serous carcinomas

\begin{tabular}{|c|c|c|c|c|c|c|c|c|}
\hline Patient & $S T I C$ & p53 (\%) & Ki-67 (\%) & $p 16(\%)$ & $R s f-1$ & Cyclin E (\%) & FASN & Muc-4 \\
\hline 1 & STIC-1 & 100 & 70 & 100 & 1 & 25 & 2 & na \\
\hline 1 & STIC-2 & 100 & 70 & 100 & 1 & 85 & 3 & na \\
\hline 1 & STIC-3 & 100 & 70 & 100 & 1 & 90 & 2 & na \\
\hline 2 & STIC-1 & 90 & 10 & 50 & 2 & 90 & 0 & -2 \\
\hline 2 & STIC-2 & 70 & 50 & 30 & 2 & 20 & 0 & -1 \\
\hline 2 & STIC-3 & 100 & 50 & 90 & 2 & 90 & 1 & 0 \\
\hline 2 & STIC-4 & 100 & 20 & 20 & 2 & 0 & 2 & na \\
\hline 2 & STIC-5 & 90 & 3 & 0 & 2 & 0 & 2 & na \\
\hline 2 & STIC-6 & 60 & 20 & 10 & 2 & 0 & 2 & na \\
\hline 2 & CA & 100 & 60 & 10 & 2 & 10 & 2 & na \\
\hline 3 & STIC-1 & $100^{\mathrm{a}}$ & 8 & 0 & na & 25 & 1 & 0 \\
\hline 3 & STIC-2 & $100^{\mathrm{a}}$ & 5 & 10 & 1 & 40 & 2 & 0 \\
\hline 3 & CA & $100^{\mathrm{a}}$ & 90 & 100 & 1 & 100 & 2 & na \\
\hline 4 & STIC-1 & $100^{\mathrm{a}}$ & 5 & 50 & 0 & 30 & 1 & 0 \\
\hline 4 & STIC-2 & $100^{\mathrm{a}}$ & 20 & 40 & 0 & 85 & 1 & 0 \\
\hline 5 & STIC-1 & 70 & 8 & 0 & 0 & 0 & 0 & na \\
\hline 5 & CA & 100 & 40 & 100 & 0 & 60 & 2 & na \\
\hline 6 & STIC-1 & 0 & 50 & 0 & 1 & 30 & 0 & -1 \\
\hline 6 & CA & 0 & 40 & 0 & 1 & 20 & 0 & -2 \\
\hline 7 & STIC-1 & 100 & 30 & 0 & 2 & 100 & 2 & -2 \\
\hline 8 & STIC-1 & 80 & 30 & 5 & 2 & 80 & 2 & 0 \\
\hline 9 & STIC-1 & 0 & 10 & 50 & 1 & 100 & 2 & -1 \\
\hline 10 & STIC-1 & 90 & 40 & 100 & 2 & 100 & 0 & -3 \\
\hline 11 & STIC-1 & 100 & 70 & 90 & 2 & 100 & 2 & -2 \\
\hline 11 & CA & 100 & 70 & 100 & 2 & 100 & 2 & -2 \\
\hline 12 & STIC-1 & 0 & 10 & 20 & 1 & 0 & 0 & -2 \\
\hline 12 & CA & 0 & 6 & 5 & 1 & 100 & 1 & -2 \\
\hline 13 & STIC-1 & 100 & 80 & na & 0 & 70 & 1 & 0 \\
\hline 13 & STIC-1 & 100 & 80 & na & 0 & 70 & 1 & 0 \\
\hline 13 & CA & 100 & 50 & na & 0 & na & 2 & 0 \\
\hline 14 & STIC-1 & 100 & 90 & 90 & 0 & 35 & 2 & na \\
\hline 15 & STIC-1 & 90 & 2 & 0 & 0 & 0 & 0 & 0 \\
\hline 16 & STIC-1 & 100 & 60 & 50 & 0 & 70 & 0 & na \\
\hline 16 & STIC-2 & 90 & 40 & 70 & 1 & 0 & 1 & 0 \\
\hline 16 & CA & 100 & 80 & 30 & 1 & 20 & 2 & 1 \\
\hline 17 & STIC-1 & 0 & 80 & 10 & 0 & 0 & 1 & -1 \\
\hline 17 & CA & 0 & 85 & 25 & 0 & na & na & -1 \\
\hline 18 & STIC-1 & 100 & 7 & na & 0 & 90 & 1 & -1 \\
\hline 18 & CA & 100 & 20 & na & 0 & 95 & 1 & 0 \\
\hline 19 & STIC-1 & 100 & 30 & 100 & 0 & 0 & 0 & 0 \\
\hline 19 & STIC-2 & 100 & 80 & 100 & 0 & 40 & 0 & 1 \\
\hline 19 & STIC-3 & 0 & 10 & 20 & 0 & 0 & 0 & -1 \\
\hline 20 & STIC-1 & 100 & 2 & 10 & 1 & 100 & 0 & 0 \\
\hline 20 & CA & 100 & 80 & 100 & 3 & 80 & 2 & 0 \\
\hline 21 & STIC-1 & 90 & 20 & 0 & na & 0 & 0 & 0 \\
\hline 21 & CA & 0 & 50 & 0 & 3 & 80 & 2 & 0 \\
\hline 22 & STIC-1 & na & 50 & na & 2 & 75 & 1 & 0 \\
\hline 23 & STIC-1 & 100 & 45 & 100 & 1 & na & 0 & -1 \\
\hline 23 & STIC-2 & 100 & 75 & 100 & 1 & na & 1 & 0 \\
\hline
\end{tabular}

Abbreviation: na, not analyzed.

${ }^{\mathrm{a}}$ Faint nuclear staining. Faint staining was interpreted as negative.

The percentage of intensely immunoreactive nuclei were determined for p53, Ki-67, p16 and cyclin E, whereas a three-tier intensity score was used to compare the immunoreactivity of Rsf-1, FASN and mucin-4 in STICs to adjacent normal-appearing tubal epithelium. The final intensity score was the intensity score of the lesion minus the intensity score of normal-appearing tubal epithelium in the same specimens.

For p53, a score of 0 has been shown to correlate with a nonsense mutation. 
$H \& E$

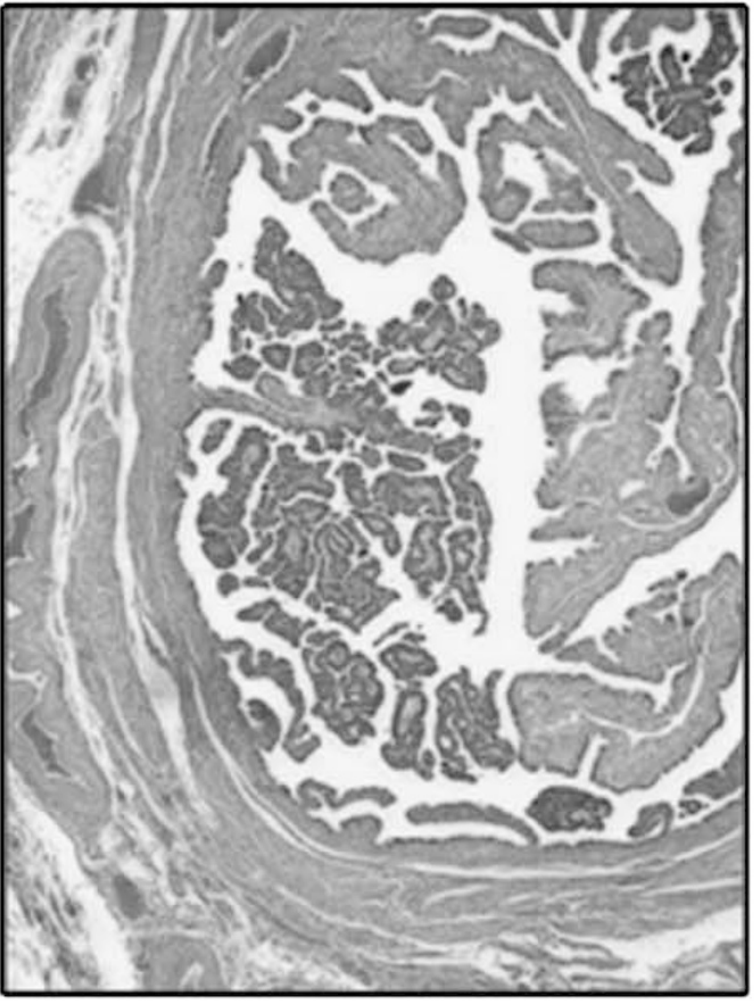

p53

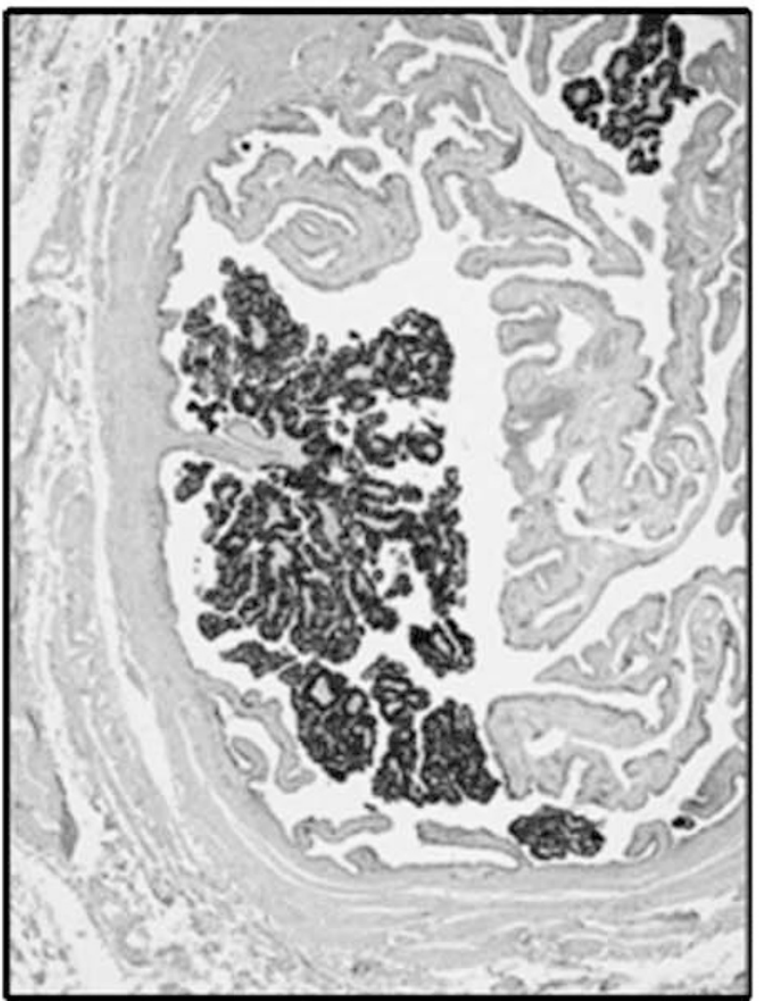

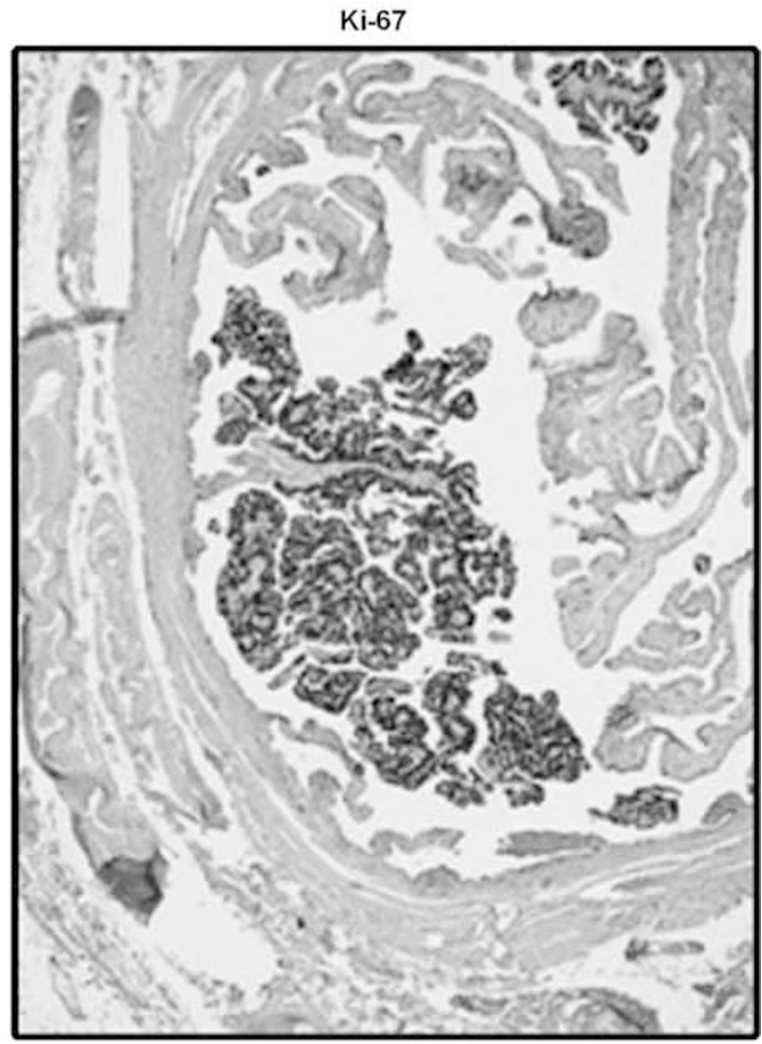

p16

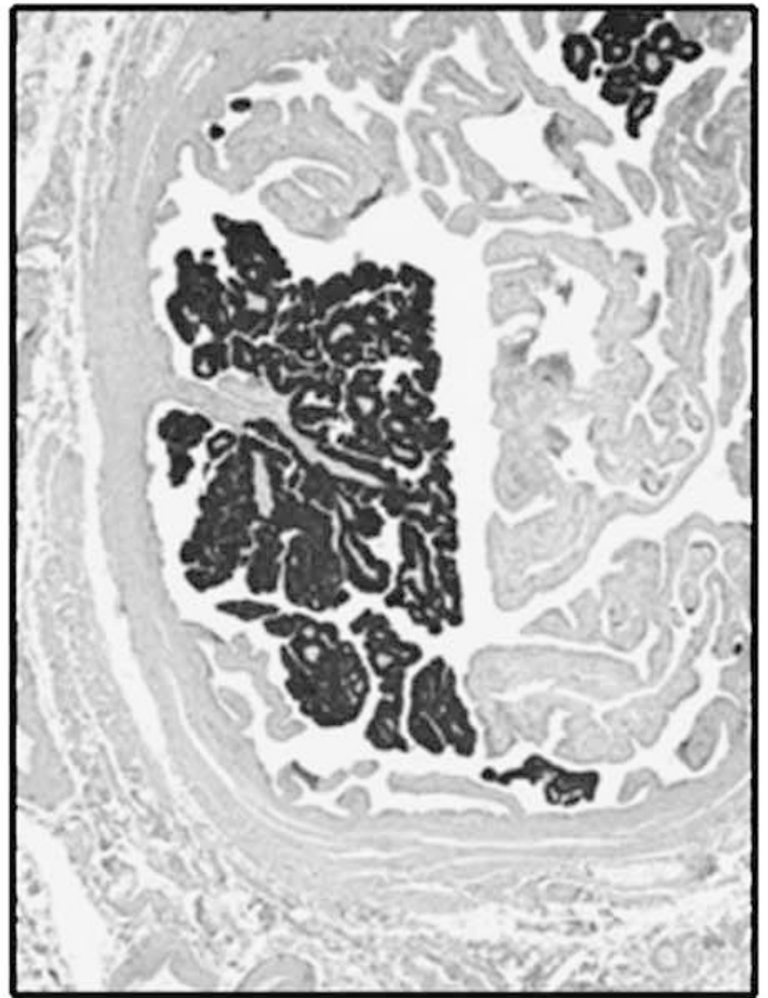

Figure 1 An example of a serous tubal intraepithelial carcinoma (STIC) (from patient 9). Hematoxylin and eosin (H\&E) stain shows a discrete papillary growth of STIC without evidence of stromal invasion. The epithelial cells of the STIC are intensely positive for p53 and p16. The Ki-67 labeling index is high (>70\%). 
immediate adjacent tubal epithelium, which showed patchy, less intense p53 nuclear staining (Figure 1). Five STICs were completely negative for p53. Thus, a total of $32(89 \%)$ of 36 STICs showed either intense p53 $(n=27)$ or negative p53 $(n=5)$ immunoreactivity. Both of these patterns are considered surrogate markers for TP53 mutations because missense mutations are almost always associated with intense nuclear stain, whereas nonsense mutations encode truncated proteins that are not recognized by the p53 antibody. In cases with multiple STICs, the p53 immunostaining pattern was identical in all STICs except in one patient (patient 19) in whom one of the three STICs was completely negative for p53, whereas the other two were intensely positive, suggesting different TP53 mutations.

\section{p16}

p16 staining was variable in the STICs. Diffuse nuclear p16 immunoreactivity was observed in 18 (55\%) of 33 STICs (Figures 1 and 2). p16 nuclear immunoreactivity was not detected in normalappearing tubal epithelium.

\section{Ki-67}

The percentage of Ki-67 positive cells was generally low and varied in different regions of normalappearing tubal epithelium. To determine a cut-off value for the Ki-67 labeling index (percentage of Ki67 positive cells) that corresponded to the level of proliferation in normal fallopian tubes, we stained 15 normal fallopian tubes, which were removed for benign reasons. We found that the mean plus 1 s.d. of the Ki-67 labeling index in normal fallopian tubes was $2.5 \% \pm 0.8 \%$. Therefore, we used an arbitrary cutoff of $10 \%$ to define an elevated Ki-67 labeling index in this study. Using this cutoff, an elevated Ki-67 labeling index $(\geq 10 \%)$ was observed in 29 $(78 \%)$ of 37 STICs. All the markers were expressed in STICs with very low Ki-67 proliferation indices $(<10 \%)$. In STICs with a higher Ki-67 index $(\geq 10 \%)$, the number expressing either diffuse nuclear p53 or showing complete absence of immunoreactivity for p53 was significantly increased ( $P=0.028$, Fisher's exact test) (Table 2). As noted earlier, the total STICs that were available for immunostaining varied as some small STICs were completely depleted during sectioning and, therefore, were not available for immunohistochemical analysis. Of particular interest were the immunostaining patterns in eight cases in which the Ki-67 proliferation index in the STICs was very low $(<10 \%)$ (Table 2$)$. The p53 staining pattern was consistent with a p53 mutation $(\geq 50 \%$ positive cells or completely negative) in $63 \%$ of STICs and Rsf- 1 was expressed in $43 \%$ and both cyclin $E$ and FASN in $63 \%$.

\section{Rsf-1}

Normal tubal epithelium expresses Rsf-1 at a low level. Increased Rsf-1 immunoreactivity compared with that expressed in normal-appearing tubal epithelium occurred in $22(63 \%)$ of 35 STICs (Figure 2). Fifteen (75\%) of 20 STICs with elevated Rsf-1 expression were also positive for cyclin E. Similarly, 15 (68\%) of 22 STICs with elevated Rsf-1 expression showed increased immunoreactivity of FASN.

\section{FASN}

Normal tubal epithelium (secretory cells) expresses FASN but FASN was increased in $23(62 \%)$ of 37 STICs compared with adjacent normal tubal epithelium (Figure 3).

\section{Cyclin E}

Cyclin E nuclear staining was seen in 24 (77\%) of 35 STICs while normal-appearing tubal epithelial cells were all negative.

\section{Mucin-4}

When compared with normal-appearing tubal epithelium, only 1 of 28 STICs showed increased mucin-4 immunoreactivity, whereas $12(43 \%)$ and $15(54 \%)$ of 28 STICs showed decreased or no change in staining, respectively (Figure 4; Table 1). To further address the expression pattern of mucin-4 in fallopian tubes and ovarian surface epithelium from individuals without carcinoma, we performed mucin-4 immunohistochemistry on eight pairs of normal fallopian tubes and ovaries. Mucin-4 immunoreactivity was focally positive in tubal epithelium but not in ovarian surface epithelium (data not shown).

\section{Comparison of Immunohistochemistry of Multiple STICs With or Without Associated Ovarian Serous Carcinomas}

Of the 12 invasive carcinomas, 4 did not express increased Rsf-1 immunoreactivity, 2 did not express p16 and one of these two also failed to express increased FASN. Aside from these, all informative invasive carcinomas expressed all of the markers. In the 12 cases in which there were concomitant invasive carcinomas with STICs, the marker pattern was concordant in 5 and discordant in 7 (Table 3). Among these discordant cases, one (patient 21) contained an STIC in which the p53 was positive in $90 \%$ of the tumor cells and an associated carcinoma in which the p53 was completely negative, suggesting that the STIC and the carcinoma had different TP53 mutations. In addition, discordant patterns were observed in 2 cases (patients 19 and 23) with 
a
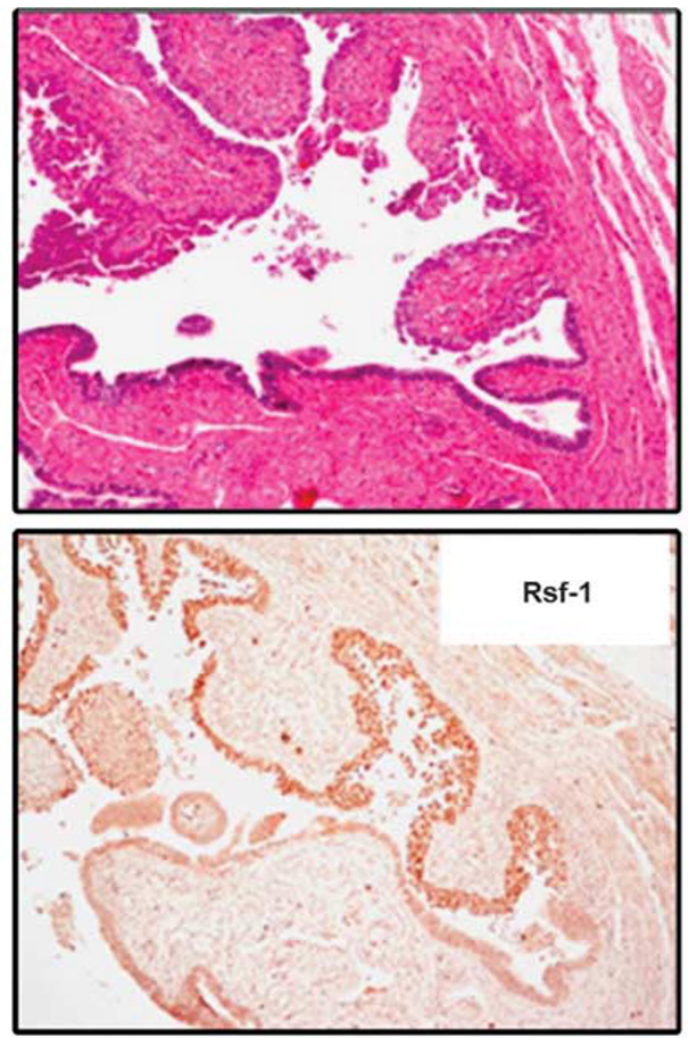

b
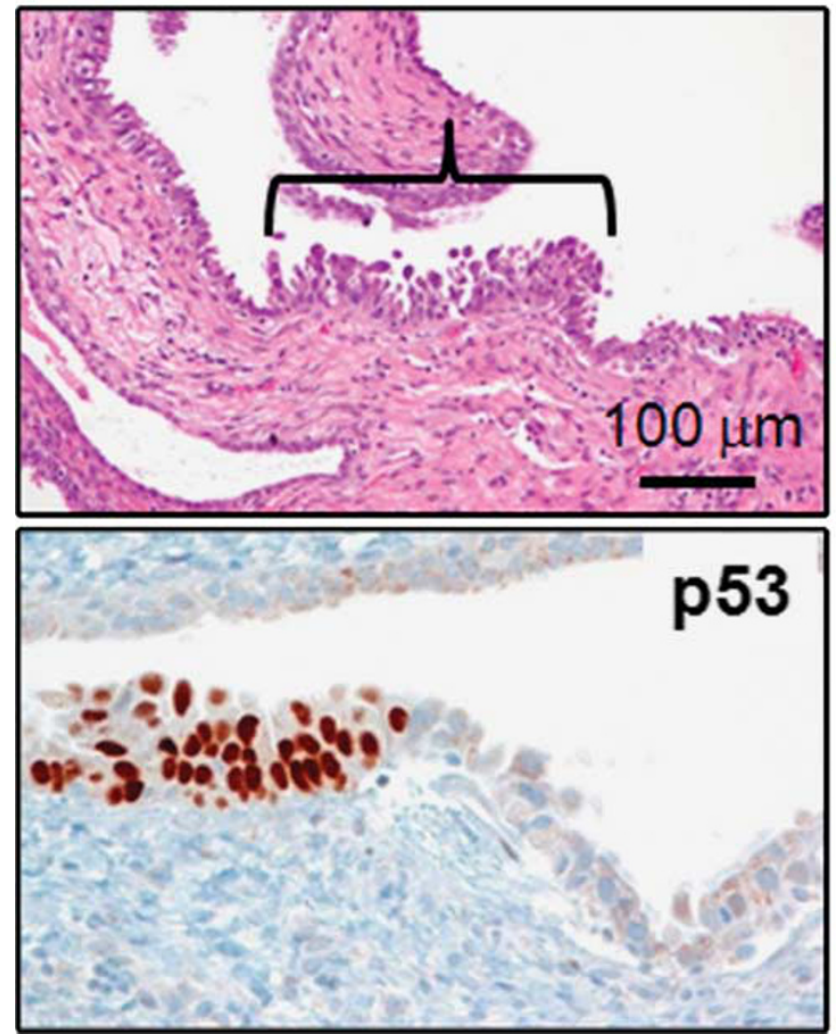
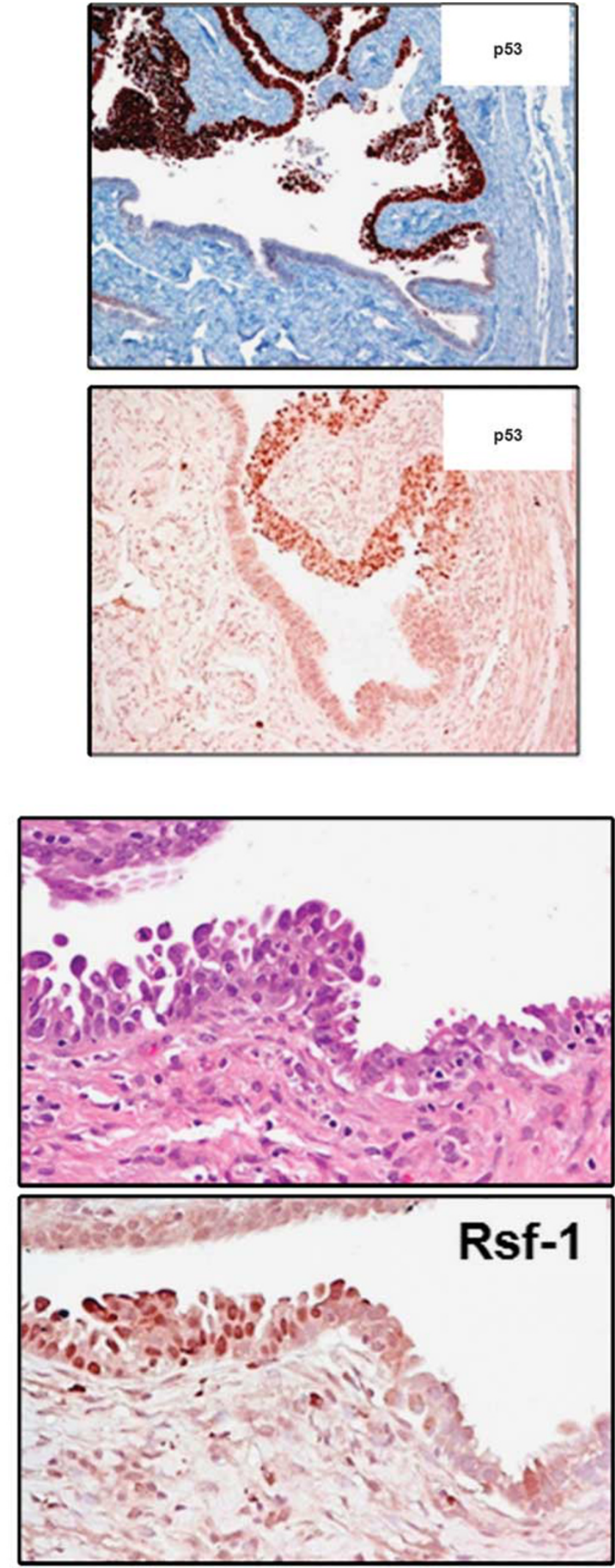

Figure 2 Rsf-1 upregulation in two representative STICs (a and b). (a) A relatively large STIC (patient 7). (b) A small STIC (patient 23, STIC-1). Rsf-1 immunoreactivity is more intense in the epithelial cells within both STICs than in the adjacent normal-appearing tubal epithelium and parallels p53 expression. 
Table 2 Increased marker expression in STICs correlated with Ki-67 proliferation index

\begin{tabular}{|c|c|c|c|c|c|c|}
\hline Ki-67 index & No. of cases & p53 & $p 16$ & $R s f-1$ & Cyclin $E$ & FASN \\
\hline$<10 \%$ & 8 & $5 / 8(63 \%)$ & $3 / 7(43 \%)$ & $3 / 7(43 \%)$ & $5 / 8(63 \%)$ & $5 / 8(63 \%)$ \\
\hline $10-49 \%$ & 14 & 13/14 (93\%) & $6 / 14(43 \%)$ & 10/13 (77\%) & $6 / 13(46 \%)$ & 7/14 (50\%) \\
\hline$\geq 50 \%$ & 15 & $14 / 14(100 \%)$ & $9 / 12(75 \%)$ & $9 / 15(60 \%)$ & $13 / 14(93 \%)$ & $11 / 15(73 \%)$ \\
\hline Total & 37 & $32 / 36(89 \%)$ & $18 / 33(55 \%)$ & $22 / 35(63 \%)$ & $24 / 35(77 \%)$ & $23 / 37(62 \%)$ \\
\hline
\end{tabular}

For p53, a case was considered positive if the intensity score was 0 or $\geq 50 \%$ (intense nuclear), as both of these patterns are associated with TP53 mutations. Increased expression for p16 was defined as $\geq 50 \%$ of positive cells, for cyclin $\mathrm{E} \geq 10 \%$ of positive cells, for Rsf- $1 \geq 1$ (final score) and for FASN $\geq 1$ (final score). The final score for Rsf-1 and FASN was determined by subtracting the intensity of the stain in normal adjacent tubal epithelium from the initial intensity score in the STIC.

multiple STICs but in which there was not an associated carcinoma. In one of these cases (patient 19) with three separate STICs, p53 was expressed in $100 \%$ of cells in 2 STICs and in none of the cells in the third STIC, suggesting that the last STIC had a different mutation from the other two. In another case of multiple STICs without an associated carcinoma (patient 23), one STIC expressed increased FASN expression and the other did not. Cyclin E and FASN were more frequently expressed in high-grade serous carcinomas than in STICs. One case (in patient 11) was of particular interest. A stretch of epithelium measuring $\sim 1 \mathrm{~mm}$ located at the margin of an STIC showed cytologic atypia that was not as marked as in the STIC. It was diffusely p53 positive but the Ki-67 proliferation index was not elevated (Figure 5). This lesion may represent a potential precursor of an STIC, where nuclear atypia and probably a TP53 mutation occurred before an aberration in cell cycle progression. We have tentatively designated this lesion as 'serous tubal intraepithelial lesion' (STIL). Rsf-1 and cyclin E proteins were overexpressed in this lesion, whereas FASN upregulation was largely confined to the STIC. Mucin-4 immunoreactivity showed a reverse pattern in which mucin-4 immunoreactivity was decreased in the STIC compared with the STIL and the normal-appearing tubal epithelium.

\section{Discussion}

The findings in this study indicate that in addition to TP53, several genes including Rsf-1, cyclin E, p16 and FASN that are amplified or upregulated in highgrade ovarian serous carcinoma are also overexpressed in the majority of STICs. In contrast, mucin-4 expression was either downregulated or showed no change in most STICs. Approximately $90 \%$ of STICs displayed intense or virtually no p53 immunoreactivity. Both of these immunostaining patterns are highly associated with TP53 mutations. A complete absence of p53 immunoreactivity has been shown in these lesions and in high-grade serous carcinoma to be associated with nonsense TP53 mutations that result in a truncated protein, which is not recognized by the p53 antibody.
Rsf-1, also known as HBXAP, was upregulated in $63 \%$ of STICs. It encodes a nuclear protein, ${ }^{14}$ which is a subunit in a chromatin assembly factor, called ISWI-containing factor RSF. ${ }^{15}$ Rsf-1 has been shown to function as a histone chaperone, whereas its binding partner, hSNF2H, possesses nucleosomedependent ATPase activity. ${ }^{16}$ The Rsf-1/hSNF2H complex (RSF complex) mediates ATP-dependent chromatin remodeling by mobilizing nucleosomes, a process that is essential for transcriptional activation or repression, ${ }^{17}$ DNA replication ${ }^{18}$ and cell cycle progression. ${ }^{19}$ In ovarian cancer, $R s f-1$ amplification has been detected in $\sim 15 \%$ and Rsf- 1 overexpression in the majority of high-grade serous carcinomas. $^{8,12,20}$ It seems that in these serous carcinomas, cellular proliferation and survival depend on the expression of Rsf-1, especially in the presence of paclitaxel. ${ }^{8,21,22}$ In addition, Rsf-1 amplification or upregulation is associated with shorter overall survival in patients with high-grade serous carcinoma patients when compared with tumors in which Rsf-1 is not amplified or upregulated. ${ }^{8}$

Cyclin E was expressed in 77\% of STICs. In all, 20 $(83 \%)$ of 24 STICs that expressed cyclin E showed either intense or no p53 staining. Cyclin E that is encoded by CCNE1 is a well-established cell cycle protein that complexes with $\mathrm{Cdk} 2$ to mediate the transition from $\mathrm{G} 1$ to $\mathrm{S}$ phase by inactivating the retinoblastoma protein. Cyclin $\mathrm{E}$ expression is upregulated in a variety of neoplasms through amplification of the CCNE1 or through its transcriptional activation. Among amplicons in high-grade serous carcinomas, the CCNE1 locus is the most common, occurring in $\sim 36 \%$ of specimens. ${ }^{20}$ Cyclin $\mathrm{E}$ has been shown to have a function in the pathogenesis of ovarian carcinoma, ${ }^{9,23-25}$ and therefore the high proportion of STICs expressing cyclin $\mathrm{E}$ underscores the important function of cyclin $\mathrm{E}$ in the early events of high-grade serous carcinogenesis.

FASN was expressed in $62 \%$ of STICs. In all, 19 $(86 \%)$ of 22 STICs that overexpressed FASN showed either intense or no p53 staining. FASN encodes a cytoplasmic enzyme that is responsible for biochemical processes involving de novo fatty acid synthesis. ${ }^{26}$ As normal adult tissues contain abundant dietary lipids, only a minimal amount of FASN is expressed. In contrast, in a variety of neoplastic 

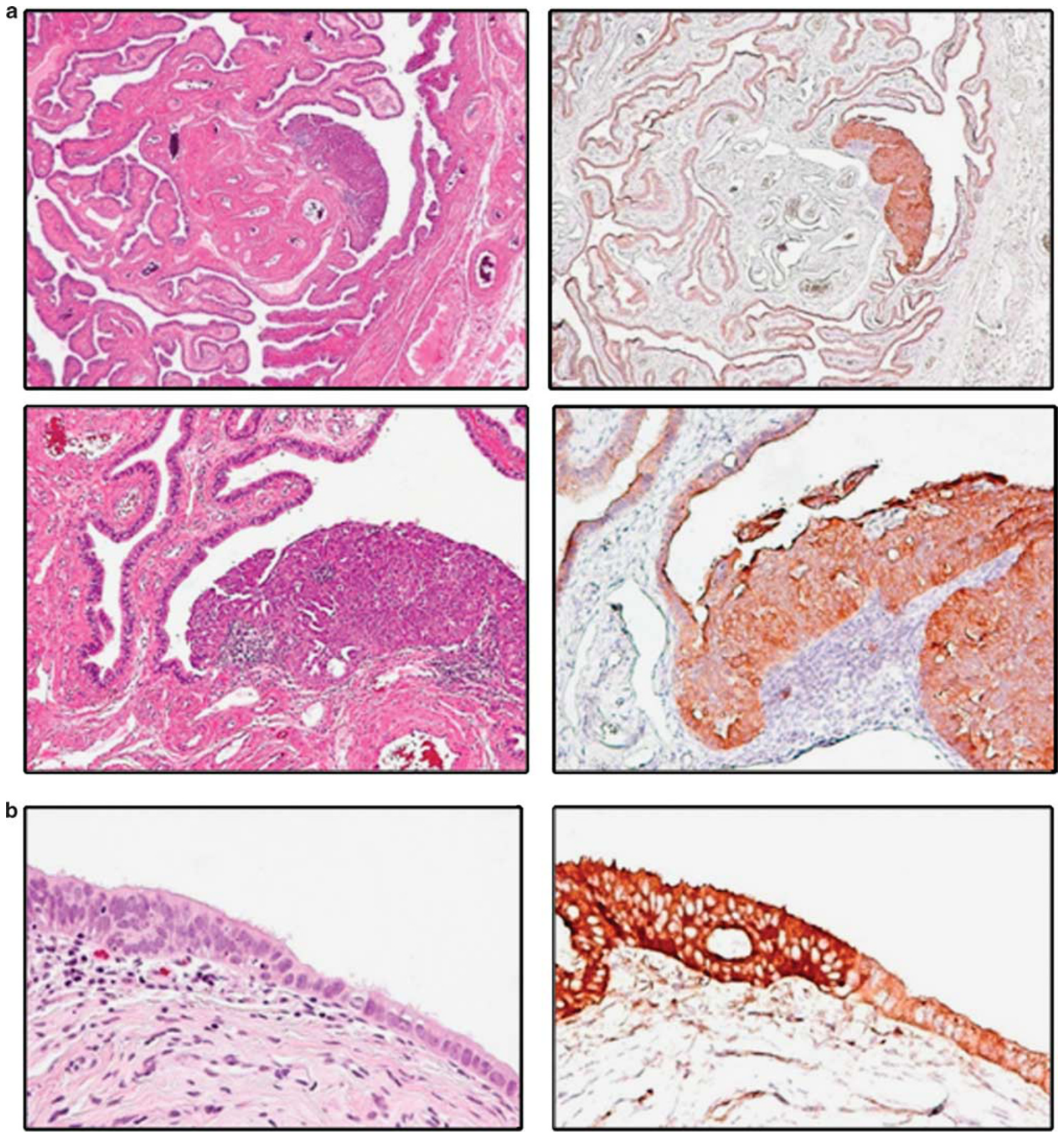

Figure 3 Overexpression of FASN in STICs. (a) A large STIC (patient 9). (b) A small STIC (patient 1, STIC-2). For both cases, FASN immunoreactivity in the STIC $(3+)$ is significantly higher than that in the normal-appearing tubal epithelium $(1+)$.

diseases including ovarian serous carcinomas ${ }^{13,26,27}$ elevated FASN levels are detected. In mouse xenograft ovarian tumor models that overexpress FASN, inhibition of the enzyme activity led to a reduction in tumor volume. ${ }^{10}$ Although the mechanism by which FASN participates in tumor progression remains unclear, it has been proposed that de novo synthesis of long chain fatty acid is important to promote tumor growth and survival. In addition,
FASN may act in concert with other signaling pathways including AMP-activated kinase, AKT and ErbB2 $2^{28-31}$ to mediate tumor initiation and progression.

In this study, $89 \%$ of STICs showed immunostaining patterns consistent with mutations of TP53 confirming that TP53 mutations are highly prevalent in STICs with a mutation frequency similar to highgrade serous carcinomas. Our data also show that 
a

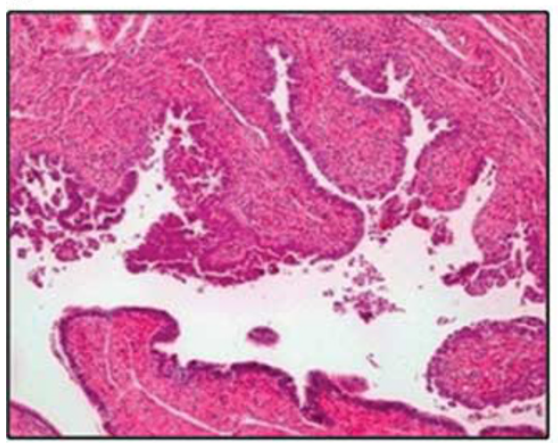

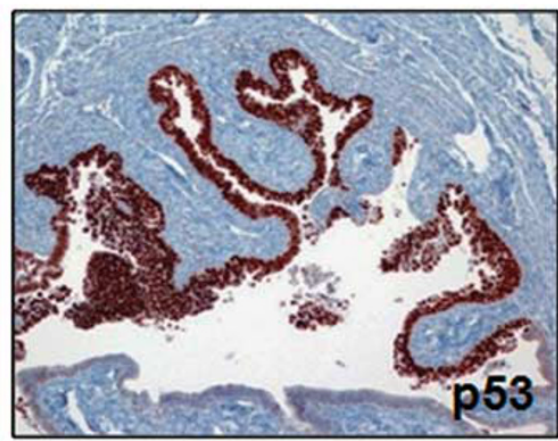

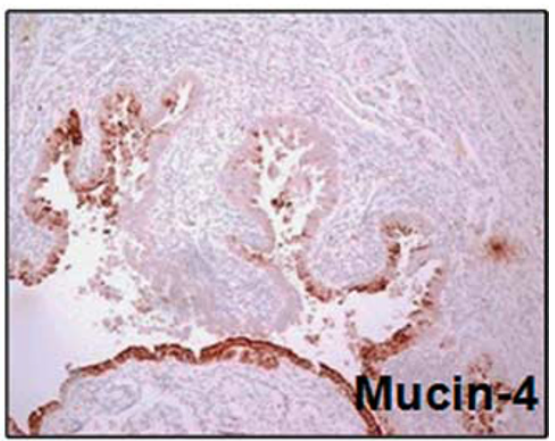

b
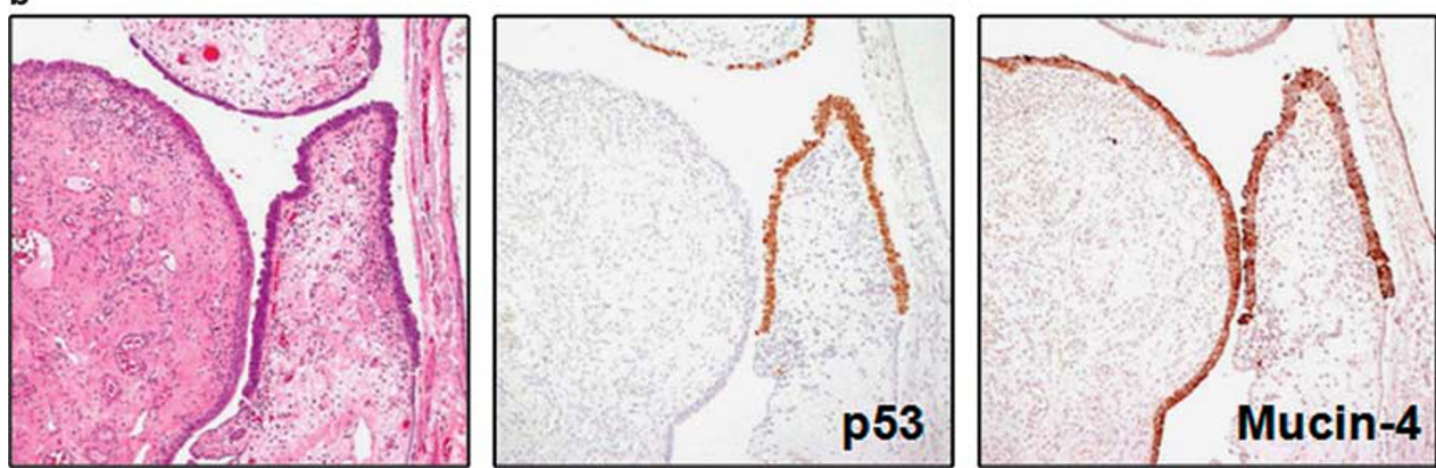

Figure 4 Expression of mucin-4 in STICs. (a) Mucin-4 immunoreactivity is largely lost in a large STIC (patient 10). (b) The intensity of the immunostain in the small STIC (patient 13, STIC-1) is the same as in the adjacent tubal epithelium.

Table 3 Discordant expression patterns in cases with multiple STICs and/or an associated carcinoma

\begin{tabular}{|c|c|c|c|c|c|c|}
\hline Patient & Lesions & p53 & $p 16$ & $R s f-1$ & Cyclin E & FASN \\
\hline \multirow[t]{7}{*}{2} & STIC-1 & & & & & \\
\hline & STIC-2 & & & & & \\
\hline & STIC-3 & & & & & \\
\hline & STIC-4 & & & & & \\
\hline & STIC-5 & & & & & \\
\hline & STIC-6 & & & & & \\
\hline & CA & & & & & \\
\hline \multirow[t]{3}{*}{3} & STIC-1 & & & & & \\
\hline & STIC-2 & & & & & \\
\hline & CA & & & & & \\
\hline \multirow[t]{2}{*}{5} & STIC-1 & & & & & \\
\hline & CA & & & & & \\
\hline \multirow[t]{2}{*}{12} & STIC-1 & & & & & \\
\hline & CA & & & & & \\
\hline \multirow[t]{3}{*}{16} & STIC-1 & & & & & \\
\hline & STIC-2 & & & & & \\
\hline & CA & & & & & \\
\hline \multirow[t]{2}{*}{20} & STIC-1 & & & & & \\
\hline & CA & & & & & \\
\hline 21 & $\begin{array}{l}\text { STIC-1 } \\
\text { CA }\end{array}$ & & & & & \\
\hline
\end{tabular}

Filled box: p53 $=0$ or $\geq 50 \%$ (intense nuclear); p16 $\geq 50 \%$; cyclin $\mathrm{E} \geq 10 \% ;$ Rsf-1 $\geq 1$ (net score); FASN $\geq 1$ (net score). these p53 staining patterns were substantially higher than the other markers examined in this study (Table 2). In other words, several STICs show either intense or no p53 staining but did not show upregulation of p16, Rsf-1, cyclin $\mathrm{E}$ and FASN (Table 1). Moreover, analysis of eight STICs with very low proliferation indices (Ki-67 labeling $<10 \%$ ) showed that most of these lesions displayed p53 immunostaining patterns consistent with TP53 mutations, whereas $43 \%$ expressed increased Rsf-1 immunoreactivity. All of the associated carcinomas in this study expressed increased levels of the markers including Rsf-1, cyclin E and FASN. These findings indicate that TP53 mutations probably represent a very early molecular genetic change in the development of high-grade serous carcinoma. It is likely that TP53 mutation, as it is a gatekeeper that regulates transformation, initiates a cascade of molecular changes that allow cells to survive in the presence of DNA damage and oncogenic activation without undergoing p53-dependent senescence or apoptosis. $^{32}$

The newly proposed hypothesis of the tubal as opposed to the ovarian surface epithelial (OSE) origin of pelvic serous carcinomas challenges many of the earlier reports showing 'overexpressed' ovarian cancer-associated genes in which the expression levels in the carcinomas were almost always compared with ovarian surface epithelium. Low molecular weight forms of cyclin $\mathrm{E}$ have been reported to be reliable markers for the distinction of 

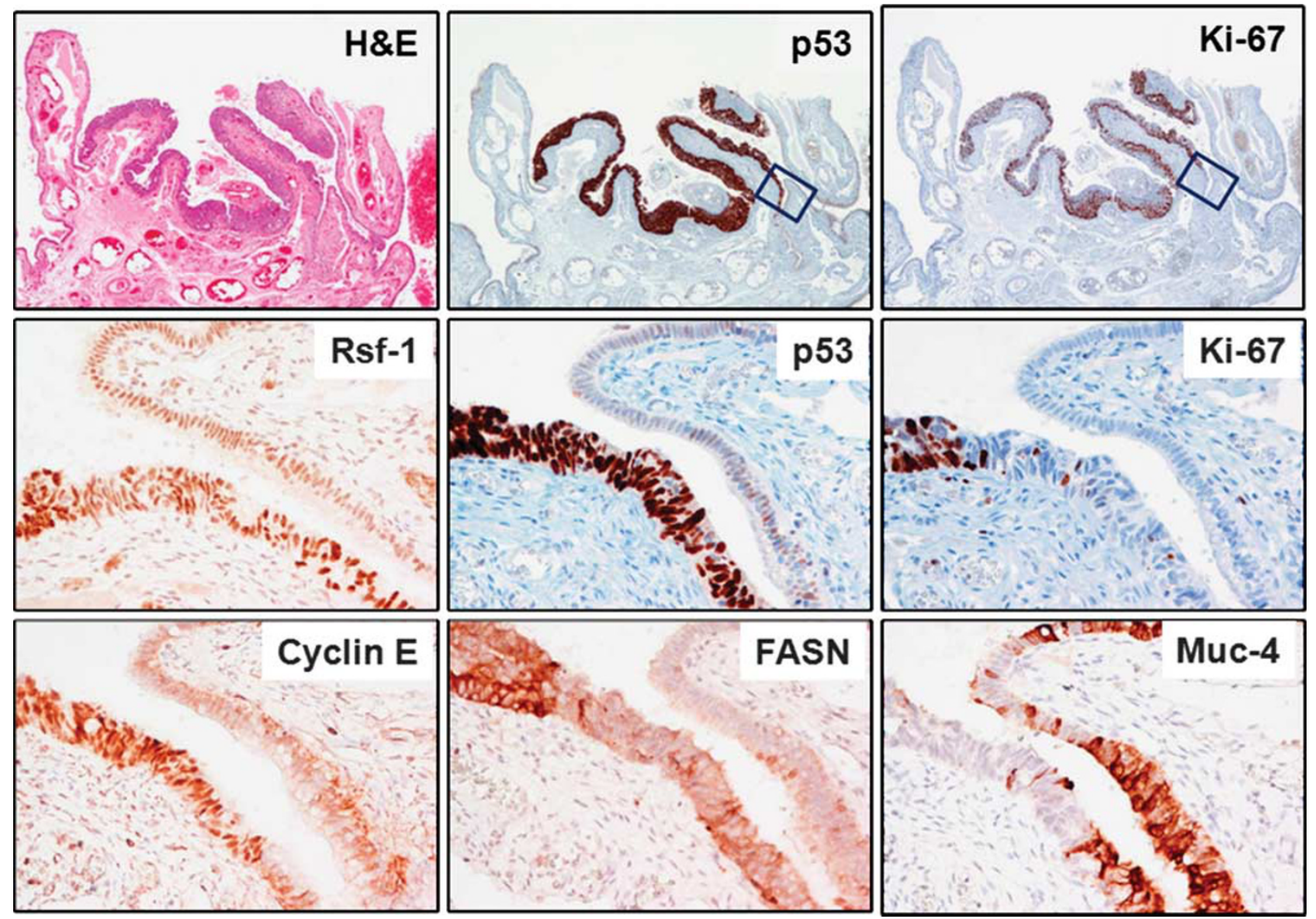

Figure 5 Immunoreactivity of p53, Ki-67, Rsf-1, cyclin E, FASN and mucin-4 in an STIC located in the fimbria (patient 11). Upper panel shows the STIC at low magnification. The epithelial cells at the right margin of the STIC show intense p53 expression but lack Ki-67 immunoreactivity (boxes). In the middle and lower panels, the increased Rsf-1 and cyclin E immunoreactivity is confined to the same region (intense p53 expression but no Ki-67 immunoreactivity). In contrast, FASN is overexpressed only in the region where there is strong p53 expression and an elevated Ki-67 proliferation index. Mucin-4 immunoreactivity (lower panel) is largely absent in the STIC but positive in the normal tubal epithelium.

ovarian carcinoma from benign and malignant mesothelial lesions. ${ }^{9}$ Accordingly, the high frequency of cyclin E expression in STICs and highgrade ovarian serous carcinoma casts further doubt on the OSE as the site of origin of these tumors. Furthermore, as the gene expression profiles in ovarian surface epithelium, which is of mesothelial origin, are different from the mullerian-derived fallopian tube epithelium, it will be important to validate whether these previously reported overexpressed genes are indeed upregulated using fallopian tube epithelium rather than OSE as a control. ${ }^{33}$ For example, mucin-4 is regarded as a biomarker of ovarian cancer, but in this study, we found that mucin-4, unlike Rsf-1, cyclin $\mathrm{E}$ and FASN, was not overexpressed in the majority of STICs when compared with the adjacent normal-appearing tubal epithelium. In fact, most STICs either retained the same level of mucin-4 immunoreactivity as the adjacent normal-appearing tubal epithelium or exhibited decreased mucin-4 expression, suggesting that mucin-4 expression is tissue lineage specific rather than tumor specific.
These findings argue against the function of mucin-4 in the pathogenesis of early high-grade serous carcinogenesis.

In conclusion, this report shows that in addition to p53 several other oncogenic proteins associated with high-grade serous carcinomas are upregulated in STICs. In this study, 95\% of STICs that overexpressed Rsf-1 showed either intense or no p53 staining, suggesting that TP53 mutations are required for Rsf-1 upregulation. The upregulation of Rsf-1 in STICs compared with the adjacent normalappearing tubal epithelium suggests that excessive RSF (Rsf)-mediated chromatin remodeling occurs early in the development of high-grade serous carcinoma. Thus, it seems that overexpression of Rsf-1, cyclin E and FASN acts in concert with TP53 mutations to propel tumor development. We also found that STICs rarely showed upregulation of mucin-4, which has been reported to be a tumorassociated protein in ovarian cancer, arguing against the function of mucin-4 in the pathogenesis of highgrade serous carcinoma. This finding underscores the importance of reassessing the validity of other 
previously reported overexpressed genes in ovarian high-grade cancer-associated proteins in which ovarian surface epithelium was used as a control. This has important clinical implications for the identification of candidate biomarkers for the development of screening tests and novel prevention strategies. Given the possibility that STICs that are detected in advanced cases of high-grade serous carcinoma may be different from those discovered incidentally, such as during risk reducing procedures in women with inherited BRCA1 or BRCA2 mutations, future studies should analyze the expression patterns of Rsf-1, cyclin E, FASN and mucin-4 in the STICs not associated with invasive carcinomas.

\section{Acknowledgements}

We appreciate the technical assistance of $\mathrm{Mr}$ Joe Vasoontara for immunohistochemistry. This study was supported by NIH/NCI RO1CA129080 and DoDOC080469.

\section{Disclosure/conflict of interest}

The authors declare no conflict of interest.

\section{References}

1 Crum CP, Drapkin R, Miron A, et al. The distal fallopian tube: a new model for pelvic serous carcinogenesis. Curr Opin Obstet Gynecol 2007;19:3-9.

2 Piek JM, van Diest PJ, Zweemer RP, et al. Dysplastic changes in prophylactically removed fallopian tubes of women predisposed to developing ovarian cancer. J Pathol 2001;195:451-456.

3 Paley PJ, Swisher EM, Garcia RL, et al. Occult cancer of the fallopian tube in BRCA-1 germline mutation carriers at prophylactic oophorectomy: a case for recommending hysterectomy at surgical prophylaxis. Gynecol Oncol 2001;80:176-180.

4 Shaw PA, Rouzbahman M, Pizer ES, et al. Candidate serous cancer precursors in fallopian tube epithelium of BRCA1/2 mutation carriers. Mod Pathol 2009;22: 1133-1138.

5 Kindelberger DW, Lee Y, Miron A, et al. Intraepithelial carcinoma of the fimbria and pelvic serous carcinoma: evidence for a causal relationship. Am J Surg Pathol 2007;31:161-169.

6 Tone AA, Begley H, Sharma M, et al. Gene expression profiles of luteal phase fallopian tube epithelium from BRCA mutation carriers resemble high-grade serous carcinoma. Clin Cancer Res 2008;14:4067-4078.

7 Marquez RT, Baggerly KA, Patterson AP, et al. Patterns of gene expression in different histotypes of epithelial ovarian cancer correlate with those in normal fallopian tube, endometrium, and colon. Clin Cancer Res 2005;11:6116-6126.

8 Shih Ie M, Sheu JJ, Santillan A, et al. Amplification of a chromatin remodeling gene, Rsf-1/HBXAP, in ovarian carcinoma. Proc Natl Acad Sci USA 2005; 102:14004-14009.

9 Davidson B, Skrede M, Silins I, et al. Low-molecular weight forms of cyclin E differentiate ovarian carcinoma from cells of mesothelial origin and are associated with poor survival in ovarian carcinoma. Cancer 2007;110:1264-1271.

10 Pizer ES, Wood FD, Heine HS, et al. Inhibition of fatty acid synthesis delays disease progression in a xenograft model of ovarian cancer. Cancer Res 1996;56: 1189-1193.

11 Davidson B, Baekelandt M, Shih IeM. MUC4 is upregulated in ovarian carcinoma effusions and differentiates carcinoma cells from mesothelial cells. Diagn Cytopathol 2007;35:756-760.

12 Mao TL, Hsu CY, Yen MJ, et al. Expression of Rsf-1, a chromatin-remodeling gene, in ovarian and breast carcinoma. Hum Pathol 2006;37:1169-1175.

13 Gansler TS, Hardman III W, Hunt DA, et al. Increased expression of fatty acid synthase (OA-519) in ovarian neoplasms predicts shorter survival. Hum Pathol 1997;28:686-692.

14 Shamay M, Barak O, Shaul Y. HBXAP, a novel PHDfinger protein, possesses transcription repression activity. Genomics 2002;79:523-529.

15 Loyola A, Huang J-Y, LeRoy G, et al. Functional analysis of the subunits of the chromatin assembly factor RSF. Mol Cell Biol 2003;23:6759-6768.

16 Aihara T, Miyoshi Y, Koyama K, et al. Cloning and mapping of SMARCA5 encoding hSNF2H, a novel human homologue of Drosophila ISWI. Cytogenet Cell Genet 1998;81:191-193.

17 Vignali M, Hassan AH, Neely KE, et al. ATP-dependent chromatin-remodeling complexes. Mol Cell Biol 2000;20:1899-1910.

18 Flanagan JF, Peterson CL. A role for the yeast SWI/SNF complex in DNA replication. Nucleic Acids Res 1999;27:2022-2028.

19 Cosma MP, Tanaka T, Nasmyth K. Ordered recruitment of transcription and chromatin remodeling factors to a cell cycle- and developmentally regulated promoter. Cell 1999;97:299-311.

20 Nakayama K, Nakayama N, Jinawath N, et al. Amplicon profiles in ovarian serous carcinomas. Int J Cancer 2007;120:2613-2617.

21 Sheu JJ, Choi JH, Yildiz I, et al. The roles of human sucrose nonfermenting protein 2 homologue in the tumor-promoting functions of Rsf-1. Cancer Res 2008;68:4050-4057.

22 Choi JH, Sheu JJ, Guan B, et al. Functional analysis of 11q13.5 amplicon identifies Rsf-1 (HBXAP) as a gene involved in paclitaxel resistance in ovarian cancer. Cancer Res 2009;69:1407-1415.

23 Courjal F, Louason G, Speiser P, et al. Cyclin gene amplification and overexpression in breast and ovarian cancers: evidence for the selection of cyclin D1 in breast and cyclin E in ovarian tumors. Int J Cancer 1996;69:247-253.

24 Farley J, Smith LM, Darcy KM, et al. Cyclin E expression is a significant predictor of survival in advanced, suboptimally debulked ovarian epithelial cancers: a Gynecologic Oncology Group study. Cancer Res 2003;63:1235-1241.

25 Marone M, Scambia G, Giannitelli C, et al. Analysis of cyclin $\mathrm{E}$ and CDK2 in ovarian cancer: gene amplification and RNA overexpression. Int J Cancer 1998;75: 34-39. 
26 Kuhajda FP. Fatty acid synthase and cancer: new application of an old pathway. Cancer Res 2006;66: 5977-5980.

27 Alo PL, Visca P, Framarino ML, et al. Immunohistochemical study of fatty acid synthase in ovarian neoplasms. Oncol Rep 2000;7:1383-1388.

28 Menendez JA, Vellon L, Mehmi I, et al. Inhibition of fatty acid synthase (FAS) suppresses HER2/neu (erbB2) oncogene overexpression in cancer cells. Proc Natl Acad Sci USA 2004;101:10715-10720.

29 Wang HQ, Altomare DA, Skele KL, et al. Positive feedback regulation between AKT activation and fatty acid synthase expression in ovarian carcinoma cells. Oncogene 2005;24:3574-3582.
30 Zhou W, Han WF, Landree LE, et al. Fatty acid synthase inhibition activates AMP-activated protein kinase in SKOV3 human ovarian cancer cells. Cancer Res 2007;67:2964-2971.

31 Grunt TW, Wagner R, Grusch M, et al. Interaction between fatty acid synthase- and ErbB-systems in ovarian cancer cells. Biochem Biophys Res Commun 2009;385:454-459.

32 Eischen CM, Weber JD, Roussel MF, et al. Disruption of the ARF-Mdm2-p53 tumor suppressor pathway in Myc-induced lymphomagenesis. Genes Dev 1999;13: 2658-2669.

33 Dubeau L. The cell of origin of ovarian epithelial tumours. Lancet Oncol 2008;9:1191-1197. 